\title{
An atypical presentation of Ormond's disease
}

\author{
João Carvão ${ }^{1, *}$ iD , Carlota Vida1,* iD, Luís Resende ${ }^{1}$ iD , Francisca Silva ${ }^{1}$ iD , Gil Silva ${ }^{1}$ \\ ${ }^{1}$ Nephrology Department, Hospital Central do Funchal, Funchal, Portugal \\ * Equally contributing first authors
}

\section{ABSTRACT}

Retroperitoneal fibrosis is a rare condition defined by an overproduction of fibro-inflammatory tissue in the retroperitoneum, usually involving the abdominal aorta, but also managing to invade adjacent structures. We report a case of a 47-year-old female patient with an atypical radiological presentation of retroperitoneal fibrosis, involving predominantly the right kidney. Ultimately, the diagnosis was performed by an immunohistochemical study that identified IgG4+ plasmocytes after kidney nephrectomy, also known as Ormond's disease. There are no universal guidelines for the treatment of retroperitoneal fibrosis, due to its rarity and lack of randomized controlled trials comparing different therapeutic strategies.

Keywords: Ormond's disease, Retroperitoneal fibrosis, End-stage kidney disease.

C 2021 Portuguese Journal of Nephrology \& Hypertension. Published by Publicações Ciência \& Vida This is an open access article under the CC BY-NC-ND license (http://creativecommons.org/licenses/by-nc-nd/4.0/).

\section{INTRODUCTION}

Retroperitoneal fibrosis (RPF) is a rare condition characterized by an overproduction of fibro-inflammatory tissue in the retroperitoneum in the absence of a dilated aorta. The tissue usually develops in the infrarenal portion of the abdominal aorta and the iliac arteries and, in late stages, involves abdominal organs or structures such as the ureters. ${ }^{1-3}$

Etiologically, it is divided into primary (idiopathic) and secondary causes. Idiopathic disease accounts for $70 \%$ of cases and can be immunoglobulin G4 (IgG4) or nonlgG4-related. Secondary RPF can result from infections, malignancy, drugs, retroperitoneal bleeding, or various other disorders. ${ }^{2}$ Idiopathic RPF is essentially a diagnosis of exclusion after secondary causes of RPF. The definitive diagnosis generally requires histopathologic confirmation by biopsy. ${ }^{4}$ Differentiation between these two forms is crucial to determine the therapeutic strategy, because in the secondary forms, it is important to solve the underlying cause. ${ }^{5}$

The clinical signs and symptoms that herald the onset of RPF are non-specific, and diagnosis is often not assumed until there is significant organ involvement. ${ }^{2}$ Ureteral obstruction causing acute or chronic renal insufficiency is a common and severe complication of RPF, and some patients may present with non-functioning kidneys as a result of long-lasting obstructive uropathy. ${ }^{1}$

Treatment of idiopathic RPF initially aims to inhibit or relieve the obstruction of the ureters or other retroperitoneal structures, using ureteric stenting or ureterolysis. However, surgical treatment does not prevent the fibro-inflammatory reaction and consequently, the progression of the disease. As such, medical therapy with corticosteroids is usually recommended in idiopathic RPF, in association or not with immunosuppressants. ${ }^{2}$

\section{CASE REPORT}

We report a case of a 47-year-old female patient that was admitted to our hospital due to anorexia and decreased urine volume. She had a past medical history of type 2 diabetes, dyslipidemia, obesity, arterial hypertension, and a stage 4 chronic kidney disease, with an atrophic left kidney revealed in a computed tomography (CT) scan performed seven years previous to this episode. On physical examination, blood pressure was $181 / 91 \mathrm{mmHg}$, with a heart rate of $74 / \mathrm{min}$, and the tympanic temperature was $37.9^{\circ} \mathrm{C}$. Neurological assessment was unremarkable. The patient had microscopic hematuria and proteinuria on urine dipstick on admission, and abnormal renal function, with a creatinine of $5.81 \mathrm{mg} / \mathrm{dL}$. The erythrocyte sedimentation rate was $88 \mathrm{~mm}$, with a white cell count of $9.6 \times 10^{\wedge} 3 / \mu \mathrm{l}$. The patient underwent an uncontrasted computer-tomography scan of her abdomen that showed a small left kidney, diffuse reduction of parenchyma thickness, and a mass with a perirenal infiltrative aspect, adhering to the right kidney, with periaortoiliac involvement and pyelocaliceal dilation as noted in Figure 1.

A ureteric stent was placed (anterograde) while pending further work-up. CT-scan guided biopsy of the perirenal tissue was performed for histological study, but the result was inconclusive. She had proteinuria of $450 \mathrm{mg} / 24 \mathrm{~h}$ and immunology revealed reduced IgG of 460mg/dL (IgG 1 3900, IgG 2 926, IgG 3 236, IgG4 229mg/L). The remaining laboratory tests, including immunology, complements, autoimmunity, and serology laboratory tests were normal (Table 1). Despite the ureteric stent, the patient's kidney function continued to worsen, and renal replacement therapy was initiated with hemodialysis.

Right radical nephrectomy was performed due to suspicious malignant disease in a patient with irreversible end-stage chronic kidney disease. Extemporaneous examination revealed a right kidney with $7.5 \times 6.2 \times 6 \mathrm{~cm}$, 


\section{Figure 1}

Uncontrasted abdomen computer tomography scan showing mass with a perirenal infiltrative aspect, adhering to the right kidney, with peri-aortoiliac involvement and pielocalicial dilation (black arrow).

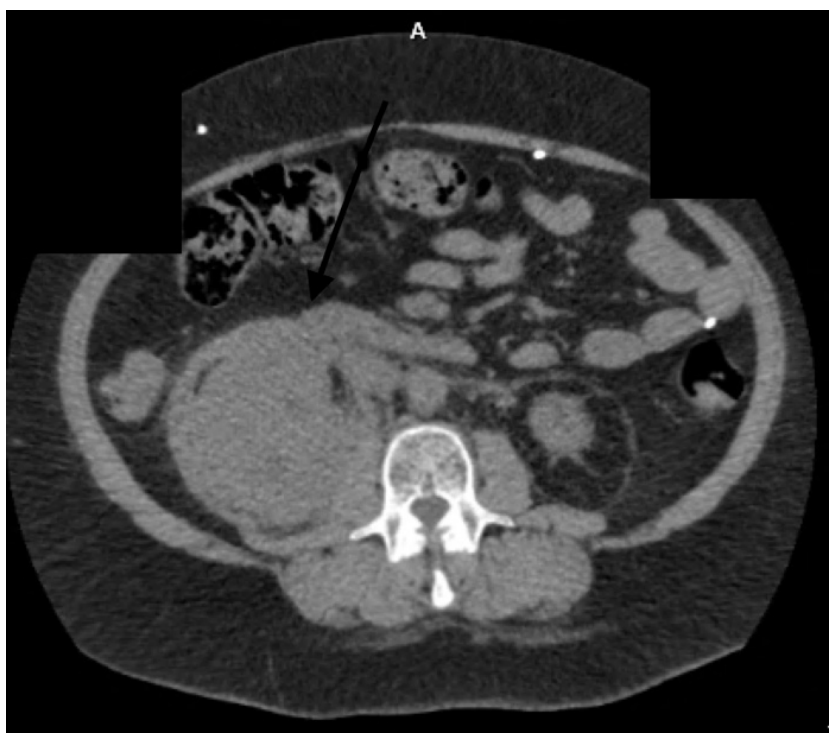

with loss of corticomedullary definition and surrounded by yellowishwhite tissue of increased consistency (Figure 2). Anatomopathological examination revealed the involvement of perirenal adipose tissue and part of the renal parenchyma by a fibro-sclerosing lesion rich in lymphoplasmacytic infiltrate with predominance of plasmocytes, presence of lymphoid aggregates, fibroblastic proliferation with dense storiform fibrosis causing parenchymal atrophy, interstitial fibrosis with tubular atrophy and involving perirenal tissue. Perineuritis and phlebitis lesions were observed. There were no signs of malignity. In the immunohistochemical study, approximately 13 to 15 IgG4+ plasmocytes per high power field were identified and an IgG4+/IgG+ plasma cell ratio of $42.9 \%$ (Figure 3). Radiologic and anatomopathological findings led us to the diagnosis of idiopathic RPF, also known as Ormond's disease, IgG4 related. However, medical therapy was not initiated due to the low likelihood of benefit in this particular case. She continues with the hemodialysis program three times a week, with no other major complications to date.

\section{DISCUSSION}

RPF is a rare disease, and its incidence ranges from 0.1 to 1.3 cases per 100,000 persons per year. It is more often seen in men, aged 40-60 years old. ${ }^{3,6,7}$ It is sometimes termed Ormond's disease, after Dr. John Ormond described it in 1948, upon observing the fibrous tissue encasement of both ureters intraoperatively in a patient with renal failure. ${ }^{8}$

RPF is part of the disease spectrum of chronic periaortitis, including inflammatory abdominal aortic aneurysm and perianeurysmal retroperitoneal fibrosis. They differ in their clinical presentation but have identical histopathological characteristics. ${ }^{9}$ However, some cases of idiopathic RPF cannot be classified as chronic periaortitis due to their

\section{$\underline{\text { Table } 1}$}

\begin{tabular}{|c|c|c|}
\hline WBC $\left(10^{\wedge} 3 / \mu l\right)$ & 9.6 & $4.2-10.8$ \\
\hline Neutrophils $\left(10^{\wedge} 3 / \mu l\right)$ & 7.0 & $1.9-7.2$ \\
\hline Hemoglobin (g/dl) & 9.2 & $11.9-14.9$ \\
\hline Platelets $\left(10^{\wedge} 3 / \mu l\right)$ & 421 & $144-450$ \\
\hline VS (mm) & 88 & $0-20$ \\
\hline Urea (mg/dl) & 353 & $8-50$ \\
\hline Creatinine (mg/dl) & 5.81 & $0.7-1.2$ \\
\hline C-reactive protein (mg/L) & 34.66 & $<6.1$ \\
\hline PTH (pg/ml) & 198 & $15-65$ \\
\hline TSH (mlU/L) & 4.84 & $0.3-4.7$ \\
\hline Free T4 (ng/dl) & 1.0 & $0.6-1.7$ \\
\hline ANA & negative & \\
\hline ANCA & negative & \\
\hline ANTI-GBM & negative & \\
\hline Anti-dsDNA & negative & \\
\hline HBsAg & negative & \\
\hline Anti-HBc & negative & \\
\hline Anti-HCV & negative & \\
\hline $\mathrm{Ag} / \mathrm{Ab} \mathrm{HIV} 1 / 2$ & negative & \\
\hline IGRA & negative & \\
\hline C3 (mg/dl) & 151 & $90-180$ \\
\hline C4 (mg/dl) & 38 & $10-40$ \\
\hline $\operatorname{lgG}(\mathrm{mg} / \mathrm{dl})$ & 460 & $700-1600$ \\
\hline $\operatorname{lgG1}(\mathrm{mg} / \mathrm{L})$ & 3900 & $4050-10100$ \\
\hline $\operatorname{lgG} 2$ (mg/L) & 926 & $1690-7860$ \\
\hline $\operatorname{lgG3}$ (mg/L) & 236 & $110-850$ \\
\hline lgG4 (mg/L) & 229 & $30-2010$ \\
\hline $\operatorname{lgA}(\mathrm{mg} / \mathrm{dl})$ & 193 & $70-400$ \\
\hline $\operatorname{lgM}(\mathrm{mg} / \mathrm{dl})$ & 65.5 & $40-230$ \\
\hline Kappa light chain (mg/dl) & 251 & $170-370$ \\
\hline Lambda light chain (mg/dl) & 132 & $90-210$ \\
\hline
\end{tabular}

WBC: white blood cells; VS: velocity sedimentation; PTH: parathyroid hormone secretion; TSH: thyroidstimulating hormone; ANA: antinuclear antibodies; ANCA: antineutrophil cytoplasmic antibodies; ANTI-GBM: Anti-glomerular basement membrane antibodies; Anti-dsDNA: Anti-double stranded DNA antibodies; HBsAg: hepatitis B virus surface antigen; Anti-HBc: hepatitis B core antibody; Anti-HCV: hepatitis C virus antibody; Ag/Ab HIV: Antigen/Antibody Human Immunodeficiency Virus; IGRA: Interferon Gamma Release Assay; IgG: immunoglobulin type G; IgA: immunoglobulin type A; IgM: immunoglobulin type $\mathrm{M}$

atypical location. Unfortunately, in our case, the abdominal CT performed did not show typical peri-renal fibrosis with ureter involvement that usually causes acute kidney injury in these patients, but it involved the right kidney predominantly. This atypical presentation led the authors to prioritize neoplastic involvement over other conditions.

HIstologic examination remains the gold standard for diagnosis. RPF is characterized histologically by a uniform proliferation of fibroblasts arranged in interlacing bundles with an infiltrative growth. ${ }^{2}$ The anatomopathological and immunohistochemical examination helped the diagnosis of IgG4-related RPF and excluded malignancy signs in our case. 


\section{Figure 2}

Right kidney with $7.5 \times 6.2 \times 6 \mathrm{~cm}$ with loss of corticomedullary definition and surrounded by yellowish-white tissue of increased consistency.

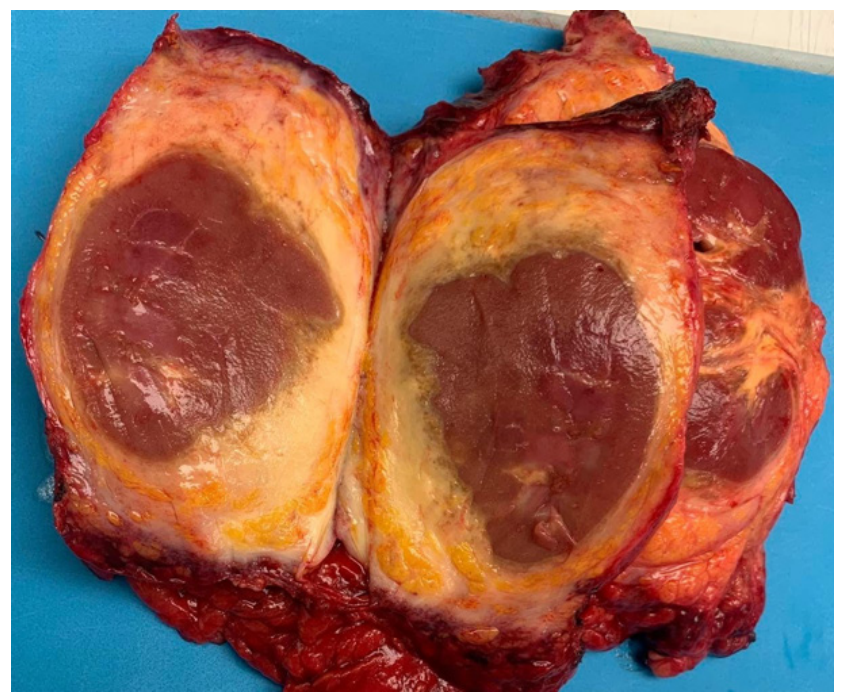

\section{Figure 3}

Left - Hematoxylin and eosin (HE) 400x: Perirenal fibro adipose tissue with lymph-plasmacytic infiltrate with plasma cells predominance; Right - Immunohistochemical study: IgG4+ plasmocytes are identified.

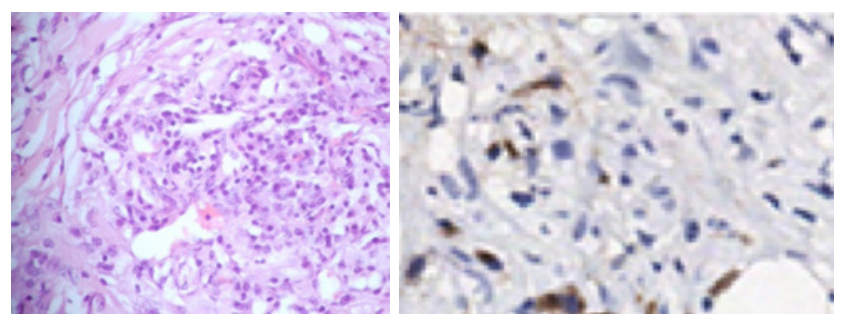

Due to the lack of prospective randomized studies and case reports, there is a lack of treatment and follow-up guidelines for these patients in the literature. Generally, there is a surgical approach which consists of the relief of ureteral obstruction with conservative procedures (such as double-J stent or nephrostomy placement). ${ }^{10,11}$ The medical approach includes treatment with glucocorticoids, sometimes associated with immunosuppressive agents, which are usually highly effective in reducing disease activity in the vast majority of patients. ${ }^{2,3}$ The recommended initial dose of prednisone is 0.5 to $1 \mathrm{mg} / \mathrm{Kg} / \mathrm{day}$, for 4 to 8 weeks, then gradually tapered to a maintenance dose of 5 to $10 \mathrm{mg} /$ day, which can be continued for 1 to 3 years. Normally, in 4 to 12 months, we can observe partial regression of the fibrosis with a control CT scan. ${ }^{11}$

Although relapses are important, to reduce long-term exposition to glucocorticoids and their side effects, other immunosuppressive agents are used in combination with prednisolone, such as methotrexate, mycophenolate mofetil, azathioprine or cyclophosphamide. The utilization of biologic agents as rituximab, in relapsing or refractory forms of disease have been tried, and the results are promising. ${ }^{11}$

Unfortunately, our patient had an end-stage kidney disease and was already on a renal replacement program, so we found no benefit in starting medical treatment, and a right radical nephrectomy was performed due to the suspicion of malignancy.

To our knowledge, there are no guidelines on the use of corticosteroid therapy in end-stage renal disease patients after removal of a large part of the inflammatory tissue. There is insufficient data regarding the prognosis and progression of the disease, mostly because published case-reports do not have sufficient follow-up. In our case, the retroperitoneal mass remains constant one year after surgery and we have not initiated corticosteroids or other immunosuppressive treatment to date.

This case illustrates an atypical radiological presentation of RPF and shows how difficult an RPF diagnosis can be. Diagnostic criteria are needed as well as new methods for the differential diagnosis between the disease's idiopathic and secondary forms.

Disclosure of potential conflicts of interest: none declared.

\section{References}

1. Corradi D, Maestri R, Palmisano A, et al. Idiopathic retroperitoneal fibrosis: Clinicopathologic features and differential diagnosis. Kidney Int. 2007;72(6):742-753.

2. Vaglio A, Salvanari C, Buzio C. Retroperitoneal fibrosi. Lancet. 2015;367:241-251.

3. Pipitone N, Vaglio A, Salvarani C. Retroperitoneal fibrosis. Best Pract Res Clin Rheumatol. 2012;26(4):439-448.

4. Khosroshahi A, Carruthers MN, Stone JH, et al. Rethinking ormond's disease: "Idiopathic" retroperitoneal fibrosis in the era of IgG4-related disease. Med (United States). 2013;92(2):82-91.

5. Runowska M, Majewski D, Puszczewicz M. Retroperitoneal fibrosis - A report of five cases. Reumatologia. 2017;55(3):140-144.

6. Van Bommel EFH, Jansen I, Hendriksz TR, Aarnoudse ALHJ. Idiopathic retroperitoneal fibrosis: Prospective evaluation of incidence and clinicoradiologic presentation. Medicine (Baltimore). 2009;88(4):193-201.

7. Vaglio A. Retroperitoneal fibrosis: New insights into clinical presentation and diagnosis. Medicine (Baltimore). 2009;88(4):208-210.

8. Ormond JK. Bilateral ureteral obstruction due to envelopment and compression by an inflammatory retroperitoneal process. J Urol. 1948;59(6):1072-1079.

9. Jois RN, Gaffney K, Marshall T, Scott DGI. Chronic periaortitis. Rheumatology. 2004;43(11):14411446.

10. Vaglio A, Maritati F. Idiopathic retroperitoneal fibrosis. J Am Soc Nephrol. 2016;27(7):1880-1889.

11. Vaglio A, Palmisano A, Alberici $F$, et al. Prednisone versus tamoxifen in patients with idiopathic retroperitoneal fibrosis: An open-label randomised controlled trial. Lancet. 2011;378(9788):338-346.

\section{ORCID}

João Carvão (iD) 0000-0003-1976-5399

Carlota Vida (iD) 0000-0003-2793-6880

Luís Resende (iD) 0000-0002-4628-6150

Francisca Silva (iD 0000-0003-3377-2368

\section{Correspondence to:}

João Nuno Jardim Carvão

Nephrology Department, Hospital Central do Funchal

Avenida Luís de Camões no 57, 9004-514 Funchal, Portugal

E-mail: jnjcarvao@campus.ul.pt 